\title{
Violencia escolar y calidad de vida relacionada con la salud en niños de escuelas primarias
}

\section{School violence and health-related quality of life in primary school children}

GARCÍA-GARCÍA, Jesús Alberto†*, OSORNIA-RAMÓN, Larissa, RAMÍREZ-CERECERO, José Ricardo y MORÁN-DELGADO, Gabriela

Universidad Autónoma de Coahuila - Facultad de Ciencia, Educación y Humanidades, México.

ID $1^{\text {er }}$ Autor: Jesús Alberto, García-García / ORC ID: 0000-0003-1369-311X, Researcher ID Thomson: D-8211-2019, CVU CONACYT ID: 568438

ID $1^{\text {er }}$ Coautor: Larissa, Osornia-Ramón

ID $2^{\text {do }}$ Coautor: José Ricardo, Ramírez-Cerecero

ID $3^{\text {er }}$ Coautor: Gabriela, Morán-Delgado / ORC ID: 0000-0001-8433-4216

DOI: $10.35429 / J B E .2020 .12 .4 .1 .7$

Recibido 10 de Julio, 2020; Aceptado 30 de Diciembre, 2020

\section{Resumen}

El objetivo de este artículo es analizar las dimensiones de la violencia escolar y su incidencia en la calidad de vida relacionada con la salud de niños de escuelas primarias en riesgo social en la ciudad de Saltillo, Coahuila. La metodología que se utilizó es cuantitativa, observacional, de corte transversal, prospectiva y descriptiva. La recopilación de la información se realizó a través de dos instrumentos, CUVE ${ }^{3}$-EP para medir la Violencia Escolar y KIDSCREEN-27 para Calidad de Vida Relacionada con la Salud, donde se tomó como muestra a 416 niños de $5^{\circ}$ y $6^{\circ}$ de Educación Primaria. El método de muestreo que se utilizó es el muestreo por conveniencia. Las técnicas estadísticas que se emplearon fueron descriptiva, correlacional y comparativa. La contribución del estudio parte de la identificación de los diferentes tipos de violencia escolar y su asociación con la calidad de vida de los niños de primaria. Siendo la violencia verbal entre los alumnos un factor que disminuye el bienestar psicológico, así como su autonomía y relación con los padres.

Violencia Escolar, Calidad de Vida Relacionada con la Salud, niños

\begin{abstract}
The objective of this article is to analyze the dimensions of school violence and its impact on the health-related quality of life of primary school children at social risk in the city of Saltillo, Coahuila. The methodology used is quantitative, observational, cross-sectional, prospective and descriptive. The information was collected through two instruments, CUVE3-EP to measure School Violence and KIDSCREEN-27 for HealthRelated Quality of Life, where 416 children aged 5 and 6 were taken as a sample. Primary education. The sampling method used is convenience sampling. The statistical techniques used were descriptive, correlational and comparative. The contribution of the study starts from the identification of the different types of school violence and its association with the quality of life of primary school children. Being verbal violence between students a factor that decreases psychological wellbeing, as well as their autonomy and relationship with parents.
\end{abstract}

School Violence, Health-Related Quality of Life, Children

Citación: GARCÍA-GARCÍA, Jesús Alberto, OSORNIA-RAMÓN, Larissa, RAMÍREZ-CERECERO, José Ricardo y MORÁN-DELGADO, Gabriela. Violencia escolar y calidad de vida relacionada con la salud en niños de escuelas primarias. Revista de Educación Básica. 2020. 4-12:1-7.

\footnotetext{
*Correspondencia al Autor (Correo Electrónico: jegarciag@uadec.edu.mx)

$\dagger$ Investigador contribuyendo como primer autor.
} 


\section{Introducción}

Los índices de violencia social a nivel mundial siguen en aumento, vivimos en una sociedad agresiva, individualista y furiosa. Los factores asociados a dicha problemática se relacionan con la cultura, la economía, política y la familia. El tema se vuelve aún más relevante, cuando la violencia trasciende al contexto escolar, debido a que en la escuela el estudiante debe aprender valores, aprender y formarse para la vida, sin embargo, muchos niños manifiestan comportamientos violentos como resultado de un entorno familiar, social y comunitario violento.

Los niños hoy en día son los más propensos a sufrir situaciones que afectan sus sentimientos, autoestima, autonomía entre otros aspectos, por ello la preocupación y el interés por resolver dichas problemáticas desde los centros educativos. Diversas circunstancias han llegado a propiciar que los niños sean más conflictivos, y que los valores cada vez se vayan perdiendo, entre ellos la falta de respeto, además de que todos estos factores han llegado afectar drásticamente a los niños en su calidad de vida puesto que están expuestos a una infinidad de condiciones que pueden ponerlos en peligro tanto a ellos como a otros, es por ello que se pretende atender a estas problemáticas para observar que aspectos de la violencia escolar repercuten en la calidad de vida relacionada con la salud de los niños.

Esta investigación analiza la incidencia de las dimensiones de la violencia escolar en la calidad de vida de niños que viven en un contexto vulnerable, lo representa un valor agregado a dicho trabajo.

Las dimensiones que fueron analizadas fueron: violencia verbal de alumnado hacia alumnado; violencia verbal de alumnado hacia profesorado; disrupción en el aula; exclusión social entre estudiantes, violencia de profesorado hacia alumnado, violencia física directa entre estudiantes y violencia física indirecta por parte del alumnado. Y las dimensiones de la calidad de vida relacionada con la salud fueron: bienestar físico, bienestar psicológico, autonomía y relación con los padres, apoyo social y compañeros, por último, el entorno escolar.
Por lo anterior, el objetivo de este artículo fue analizar las dimensiones de la violencia escolar y su incidencia en la calidad de vida relacionada con la salud de niños de escuelas primarias en riesgo social en la ciudad de Saltillo, Coahuila.

La estructura del artículo se conforma por las siguientes secciones: en primer lugar, se presenta una revisión de la literatura sobre la violencia escolar y la CVRS en población infantil, posteriormente se explica la metodología del estudio, participantes, diseño y los instrumentos utilizados. Posteriormente se presentan los resultados estadísticos para el contraste de la hipótesis central y por último se exponen las principales conclusiones del estudio.

\section{Revisión de la literatura}

La revisión de los antecedentes teóricos del tema violencia escolar, permitió identificar el estudio de Valle et al., (2019), los resultados obtenidos afirman que desde la percepción de los alumnos, los tipos de violencia dirigida al docente que se presentaron con más frecuencia fueron: la disrupción en el aula, seguida por la violencia verbal. Por lo que los alumnos son los primeros en darse cuenta de los tipos de violencia que se ejercen en el entorno escolar.

Además, Valle et al., (2019) menciona que, al analizar por sexo, las mujeres mayormente reconocieron la violencia del maestro hacia el alumno, a diferencia de los hombres. Siendo en dicho estudio las niñas más observadoras puesto que pueden llegar a identificar fácilmente conductas violentas.

Mientras que González y Treviño, (2019) encontraron que "el factor emocional fue el de mayor peso en la violencia escolar, es decir, un estudiante que no puede afrontar las cosas, será incapaz de controlar lo importante, por percibir que las dificultades se acumulan y no las puede superar, lo cual está asociado con un incremento de la violencia escolar" (pp. 135138). Se pueden distinguir la importancia de controlar las emociones para disminuir la violencia en el entorno escolar. 
Otro factor que se destacó dentro de los resultados de González y Treviño, (2019) es el "que se relacionó de manera directa con la Violencia fue Relaciones con un peso de - 0,27.

Es decir, a medida que las relaciones entre estudiantes con sus pares y/o docentes mejoran, la Violencia escolar baja". Es decir, dentro del clima escolar positivo además de los compañeros también van incluidos los docentes quienes deben establecer una relación cordial con sus alumnos e instruir en el comportamiento positivo entre compañeros, evitando que se desencadene una mayor violencia en contextos escolares.

En consideración con Sánchez et al., (2019) argumentan que "el enfoque negativo hacia la participación deportiva, la violencia experimentada y la violencia observada se correlacionan positivamente entre sí y negativamente con la responsabilidad social". Se puede percibir al deporte como negativo cuando se encuentra en relación con la violencia, mientras que cuando exista apoyo por otros no afectará.

Por otra parte los autores Valdés, Tánori, Sotelo y Ochoa, (2018) mencionan que "el acoso por pares se correlaciona de forma negativa con un clima escolar positivo". Por lo que nuevamente con dichos autores el clima escolar tiende a tener un rol indispensable en la protección de los niños cuando se enfrentan a la victimización de sus compañeros. Siendo el entorno escolar uno de los contextos más importantes ya que es donde el niño pasa la mayor parte de su tiempo, por lo que se debe buscar en todo momento que sea un lugar óptimo para los niños, donde disfruten de aprender y convivir con sus compañeros, propiciando siempre un ambiente ideal.

En cuanto Adams y Hannum, (2018) hallaron que "los estudiantes varones eran más propensos a ser víctimas de sus compañeros; ya que el ser hombre se asocia con un $51 \%$ más de probabilidades de ser víctima, en comparación con ser mujer". Por lo que en este estudio se encontró como más vulnerables al sexo masculino.
Mientras que los autores McDade, King, Vidourek y Merianos, (2018) comentan que "la comparación de género dentro de la raza no revelara diferencias significativas porque las experiencias de la raza y el clima escolar fueron las mismas para ambos géneros". Para dichos autores de acuerdo con sus resultados, tanto los hombres como las mujeres sin importar su procedencia pueden efectuar casos de violencia en la escuela.

Un dato interesante que se encontró con respecto a los alumnos es en la investigación de Deole, (2018) quien menciona que la intensidad de los actos de violencia puede aumentar a medida que los estudiantes crecen. También puede darse el caso de que los estudiantes mayores se enfrenten mejor a estos incidentes en la escuela que los estudiantes más jóvenes en los grados primarios, es por ello que aquí en México se debe trabajar desde temprana edad con los niños en temas de violencia, ya que posteriormente cuando ingresan a secundaria si dichos temas no fueron consolidados tienden aumentar, como en el caso de Estados Unidos que los niños terminan quinto grado en primaria $\mathrm{y}$ posteriormente entran a las escuelas secundarias.

\section{Metodología}

\section{Diseño}

El tipo de investigación que se llevó a cabo fue cuantitativa no experimental, observacional, transversal, prospectiva, descriptiva y comparativa (Méndez, Moreno, Namihira, y Sosa, 1990).

\section{Participantes}

Se observa que de un total de 422 sujetos el género que más predominó fue las niñas con un $50.7 \%$, mientras que los niños $47.9 \%$.

\section{Instrumentos}

Se utilizaron dos instrumentos CUVE ${ }^{3}$-EP para Violencia escolar y KIDSCREEN-27 para CVRS. 
El cuestionario CUVE ${ }^{3}$-EP consta de 36 enunciados diseñados, para evaluar la Violencia Verbal de Alumnado hacia Alumnado; Violencia Verbal de Alumnado hacia Profesorado; Disrupción en el Aula; Exclusión Social entre Estudiantes, Violencia de Profesorado hacia Alumnado, Violencia Física Directa entre Estudiantes y Violencia Física Indirecta por parte del Alumnado.

La prueba del $\mathrm{CUVE}^{3}$-EP ofrece una medida de la percepción del alumnado sobre la frecuencia de aparición de diferentes tipos de violencia en el contexto educativo.

El tiempo requerido para contestar esta prueba es de 15 a 20 minutos.

\section{KIDSCREEN- 27}

En cuanto a los instrumentos KIDSCREEN evalúan las dimensiones relevantes de la calidad de vida relacionada con la salud en niños y adolescentes de 8 a 18 años. Los instrumentos KIDSCREEN son una familia de medidas genéricas, son aplicables en diferentes contextos nacionales y culturales, cumplen con los estándares de calidad en el desarrollo de instrumentos y son prácticos (es decir, cortos y fáciles de usar).

El KIDSCREEN-27 Ravens-Sieberer et al., (2007) se desarrolló como una versión más corta del KIDSCREEN-52 con un mínimo de pérdida de información y con buenas propiedades psicométricas. Se puede utilizar en estudios epidemiológicos más amplios y también como un instrumento de detección breve entre los niños con una enfermedad crónica para quienes los problemas específicos de la enfermedad son importantes. KIDSCREEN-27 representa las diez dimensiones originales fusionadas en 5 dimensiones, lo que permite información de perfil detallada para el bienestar físico (Actividad física y salud), el bienestar psicológico (Estado de ánimo y sentimientos), la autonomía y la relación con los padres (Tu vida familiar y tu tiempo libre), el apoyo social y los compañeros (Tus amigos), el entorno escolar (La escuela y aprendizaje).
El tiempo requerido para completar el programa de computadora KIDSCREEN oscila entre 10 y 20 minutos, dependiendo de la edad del niño o adolescente. No hay límite de tiempo para completar el cuestionario.

\section{Procedimientos}

Principalmente se solicitó el permiso a los directores de cada escuela primaria, a las cuales se asistió con un oficio elaborado por la Facultad de Ciencia, Educación y Humanidades. Una vez autorizado el oficio se acudió a cada una de las escuelas que se encontraban en sectores de riesgo social de acuerdo con los resultados brindados por la Secretaria de Educación Pública, en la ciudad de Saltillo, Coahuila. La aplicación tuvo un periodo de dos semanas.

Previamente a la aplicación de las encuestas se les brindaron las instrucciones de manera general a los encargados de la aplicación, pidiendo de requisito firmar la carta de consentimiento. Posteriormente la aplicación tuvo una duración aproximada de 15 minutos, incluyendo el tiempo que tardaban los encargados en recoger cada encuesta.

\section{Análisis de resultados}

Tanto el instrumento $\mathrm{CUVE}^{3}$-EP como el KIDCREEN-27 fueron analizados mediante el paquete estadístico para ciencias sociales (SPSS) por sus siglas en inglés Statistical Package of the Social Sciences Version 21.

Teniendo el instrumento Kids-27 una serie de ítems de las versiones de KIDSCREEN los cuales cumplen con los supuestos del modelo Rasch (una dimensionalidad, homogeneidad de ítems y personas, suficiencia del puntaje total). La mayoría de los ítems están formulados positivamente y en concordancia es la puntuación, lo que significa que una puntuación más alta refleja una CVRS más alta. Para la interpretación de los puntajes de KIDSCREEN, se debe considerar el contenido de las escalas. La información básica sobre las escalas viene dada por sus definiciones.

Se realizaron análisis de frecuencias y porcentajes, estadísticos descriptivos y correlaciones. 


\section{Resultados}

Se realizó un análisis descriptivo del tema Violencia Escolar y CVRS en niños de primaria en escuelas de riesgo social ubicadas en la ciudad de Saltillo Coahuila, donde se tomaron en cuenta los estadísticos de centralidad: media, mediana y moda, a su vez se utilizó el estadístico de dispersión: desviación estándar y por último los estadísticos de distribución: curtosis y asimetría.

La dimensión que más destaca es exclusión social entre estudiantes, seguido de violencia del profesorado hacia el alumnado, y posteriormente violencia física directa $y$ amenazas entre estudiantes.

Mientras que las de menor puntaje son violencia física indirecta por parte del alumnado, disrupción en el aula, violencia verbal de alumnado hacia alumnado y violencia verbal del alumnado hacia el profesorado.

Los puntajes de la desviación estándar permiten decir que existe una estabilidad en los valores de la media.

Respecto a los estadísticos que miden la distribución de los datos se puede observar que son valores con signo positivo, esto quiere decir, que existe una tendencia hacia los valores bajos de la escala.

\begin{tabular}{|c|c|c|c|c|c|c|c|}
\hline & $\mathrm{n}$ & $\overline{\mathrm{x}}$ & Med & Mo & DE & AS & K \\
\hline $\begin{array}{l}\text { Exclusión Social } \\
\text { entre Estudiantes }\end{array}$ & 422 & 11.75 & 10 & 7 & 5.25 & 1.80 & 3.99 \\
\hline $\begin{array}{lr}\text { Violencia } & \text { del } \\
\text { Profesorado } & \text { hacia } \\
\text { el Alumnado } & \\
\end{array}$ & 422 & 11.09 & 9 & 9 & 3.60 & 2.44 & 6.48 \\
\hline $\begin{array}{lr}\text { Violencia } & \text { Física } \\
\text { Directa } & \text { y } \\
\text { Amenazas } & \text { entre } \\
\text { Estudiantes } & \\
\end{array}$ & 422 & 10.42 & 9 & 5 & 4.74 & 0.97 & 0.21 \\
\hline $\begin{array}{l}\text { Violencia Física } \\
\text { Indirecta por parte } \\
\text { del Alumnado }\end{array}$ & 422 & 9.59 & 8 & 5 & 4.56 & 1.00 & 0.23 \\
\hline $\begin{array}{l}\begin{array}{l}\text { Disrupción en el } \\
\text { Aula }\end{array} \\
\end{array}$ & 422 & 8.46 & 8 & 9 & 3.13 & 0.25 & $0.78^{-}$ \\
\hline $\begin{array}{l}\text { Violencia Verbal } \\
\text { de Alumnado } \\
\text { hacia Alumnado }\end{array}$ & 421 & 7.82 & 8 & 3 & 3.58 & 0.32 & 0.91 \\
\hline 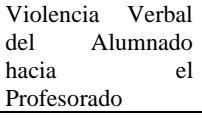 & 422 & 6.55 & 5 & 4 & 3.58 & 1.75 & 2.62 \\
\hline
\end{tabular}

Tabla 1 Análisis descriptivo del constructo Violencia Escolar
Continuando con el análisis del constructo CVRS, los puntajes más altos corresponden a las dimensiones: autonomía y la relación con los padres, seguido de bienestar psicológico. Lo que quiere decir que los niños se sienten positivos acerca de la relación con sus padres, y satisfechos con los recursos financieros que ellos les otorgan, teniendo suficiente libertad para elegir y estando emocionalmente complacidos con la vida.

Mientras que los puntajes más bajos corresponden a las dimensiones de bienestar físico, apoyo social y los compañeros, entorno escolar. Por lo que se puede decir que los niños suelen sentirse físicamente agotados, y no aptos para realizar diversas actividades, además de sentirse excluidos por sus compañeros, teniendo bajo rendimiento académico y sentimientos negativos sobre la escuela.

De acuerdo con los puntajes de la desviación estándar existe una estabilidad en los valores de la media.

Respecto a los estadísticos que miden la distribución de los datos se puede observar que todas las dimensiones están con signo negativo, lo que quiere decir, que existe una tendencia hacia los valores altos de la escala.

\begin{tabular}{|c|c|c|c|c|c|c|c|}
\hline & $\mathrm{n}$ & $\overline{\mathrm{X}}$ & Med & Mo & $\mathrm{DE}$ & AS & $\mathrm{K}$ \\
\hline $\begin{array}{l}\text { Autonomía } \\
\text { y la relación } \\
\text { con los } \\
\text { padres }\end{array}$ & 422 & 27.97 & 29 & 31 & 5.10 & -.86 & .21 \\
\hline $\begin{array}{l}\text { Bienestar } \\
\text { psicológico }\end{array}$ & 422 & 27.11 & 28 & 31 & 4.60 & -.84 & .91 \\
\hline $\begin{array}{l}\text { Bienestar } \\
\text { físico }\end{array}$ & 422 & 19.06 & 19 & 19 & 3.68 & -.48 & -.06 \\
\hline $\begin{array}{l}\text { Apoyo } \\
\text { social y los } \\
\text { compañeros }\end{array}$ & 422 & 16.77 & 18 & 20 & 3.45 & -1.46 & 2.30 \\
\hline $\begin{array}{l}\text { Entorno } \\
\text { escolar }\end{array}$ & 422 & 16.27 & 17 & 19 & 3.06 & -1.00 & 1.08 \\
\hline
\end{tabular}

Tabla 2 Análisis descriptivo del constructo de CVRS

A continuación, se muestra la correlación de Pearson donde se puede observar las relaciones entre las dimensiones de Violencia Escolar y CVRS, donde el nivel de significancia es $\mathrm{p}=0.01 \mathrm{y} \mathrm{p}=0.05$. 
El análisis de correlación de Pearson (tabla 3) nos permite identificar principalmente que existe una relación significativa inversa entre la dimensión de violencia verbal de alumnado hacia alumnado y el constructo CVRS lo que permite afirmar que:

- Niños con un menor bienestar psicológico tienen una mayor violencia verbal de alumnado hacia alumnado.

Infantes con mayor violencia verbal de alumnado hacia alumnado presentan menor autonomía y relación con los padres.

- El apoyo social y los compañeros disminuye en la medida en que crece la violencia verbal de alumnado hacia alumnado.

El entorno escolar se ve afectado en función del aumento de la violencia verbal de alumnado hacia alumnado.

Es importante observar que los niños con mayor violencia verbal de alumnado hacia alumnado presentan menor bienestar psicológico, autonomía y relación con los padres, exclusión entre compañeros y sentimientos negativos sobre la escuela. Se puede concluir que para impedir que se propicie violencia entre los niños, se debe considerar necesaria la estabilidad emocional, la cual contribuya en la autonomía del niño, generando un ambiente de cordialidad entre ellos.

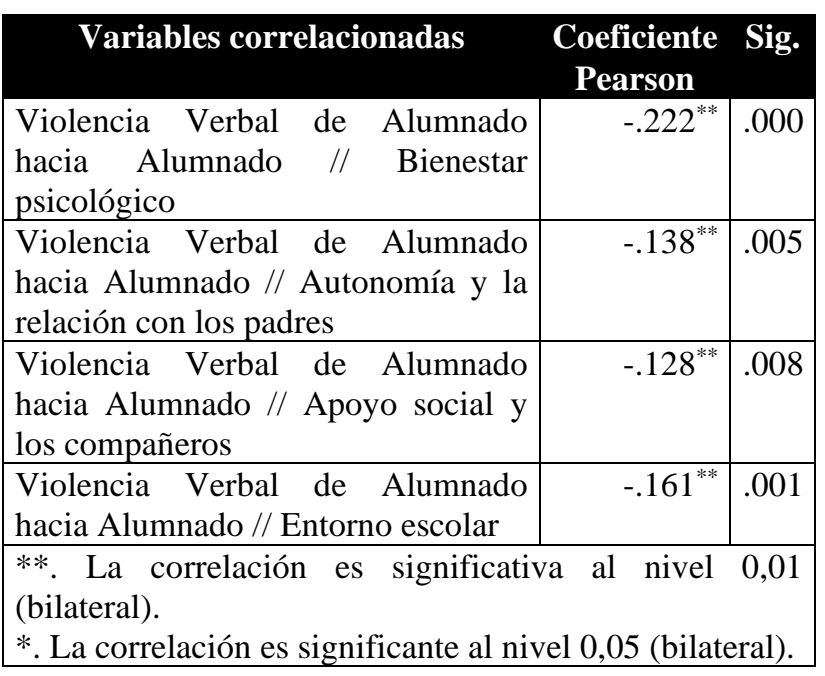

Tabla 3 Análisis correlacional de la dimensión violencia verbal de alumnado hacia alumnado y el constructo CVRS
El análisis de correlación de Pearson (tabla 4) nos permite identificar la existencia de una relación significativa inversa entre la dimensión de violencia escolar y el constructo $C V R S$ lo que permite afirmar que:

- Niños con mayor violencia verbal hacia el profesor tendrán menores niveles de Calidad de vida.

Infantes mayores disrupción en el aula presentan menor calidad de vida.

- Al aumentar la exclusión social entre estudiantes disminuirá la calidad de vida de los niños.

- Cuando aumenta la violencia del profesor hacia el alumnado disminuye la calidad de vida de los niños.

- La prevalencia de la violencia física directa se refleja en una diminución de la calidad de vida de los niños.

- La presencia de violencia física indirecta por parte del alumno se relaciona con un decremento de la calidad de vida de los niños.

\begin{tabular}{|l|r|r|}
\hline \multicolumn{1}{|c|}{ Variables correlacionadas } & $\begin{array}{c}\text { Coeficiente } \\
\text { Pearson }\end{array}$ & Sig. \\
\hline $\begin{array}{l}\text { Violencia Verbal de Alumnado } \\
\text { hacia el profesorado // CVRS }\end{array}$ & $-.153^{* *}$ & .000 \\
\hline Disrupción en el aula // CVRS & $-.105^{* *}$ & .003 \\
\hline $\begin{array}{l}\text { Exclusión social entre estudiantes // } \\
\text { CVRS }\end{array}$ & $-.298^{* *}$ & .000 \\
\hline $\begin{array}{l}\text { Violencia del profesor hacia el } \\
\text { alumnado // CVRS }\end{array}$ & $-.136^{* *}$ & .001 \\
\hline Violencia física directa// CVRS & $-.245^{* *}$ & .000 \\
\hline $\begin{array}{l}\text { Violencia física indirecta por parte } \\
\text { del alumno// CVRS }\end{array}$ & $-.216^{* *}$ & .000 \\
\hline $\begin{array}{l}* * \text { La correlación es significativa al nivel } \\
\text { (bilateral). }\end{array}$ \\
*. La correlación es significante al nivel 0,05 (bilateral). \\
\hline
\end{tabular}

Tabla 4 Análisis correlacional de las dimensiones de violencia escolar y el constructo CVRS 


\section{Conclusiones}

El entorno escolar es una variable que debe analizarse a profundidad, debido a que se encontró evidencia del impacto que tiene en la calidad de vida de los niños. Aunque estos tengan buenos niveles de apoyo familiar y bienestar psicológico, si en la escuela no encuentra un apoyo y una educación que promueva el respeto y la tolerancia, los comportamientos violentos y agresivos pueden aumentar.

Todas las dimensiones de violencia escolar tienen una incidencia en la calidad de vida de los niños, siendo el entorno escolar sano una dimensión de satisfacción y bienestar, se debe atender la problemática desde mecanismo escolares que erradiquen los comportamientos violentos, discriminación y de más expresiones de violencia.

Niños con un menor bienestar psicológico tienen una mayor violencia verbal de alumnado hacia alumnado.

Infantes con mayor violencia verbal de alumnado hacia alumnado presentan menor autonomía y relación con los padres.

El apoyo social y los compañeros disminuye en la medida en que crece la violencia verbal de alumnado hacia alumnado.

El entorno escolar se ve afectado en función del aumento de la violencia verbal de alumnado hacia alumnado.

\section{Referencias}

Adams, J., \& Hannum, E. (2018). School Violence in China: A Multilevel Analysis of Student Victimization in Rural Middle Schools. In Research in the Sociology of Education (Vol. 20, pp. 41-69). doi:10.1108/S1479353920180000020003

Deole. (2018). Human capital consequences of violence in schools: Estimating the impact of violence in schools on education outcomes in Brazil. Rev Dev Econ.
McDade, R. S., King, K. A., Vidourek, R. A., \& Merianos, A. L. (2018). Impact of Prosocial Behavioral Involvement on School Violence Perpetration Among African American Middle School and High School Students. Journal of Immigrant and Minority Health, 20(1), 7-13. doi:10.1007/s10903-016-0544-6

Méndez, I., Moreno, L., Namihira, D., \& Sosa, C. (1990). El protocolo de investigación: lineamientos para su elaboración y análisis (2a ed.). Recuperdo de: http://bibliotecasibe.ecosur.mx/sibe/book/00002 7375

González, M., \& Treviño, D. (2019). Violencia escolar en bachillerato: algunas estrategias para su prevención desde diferentes perspectivas. Revista Interuniversitaria. Recuperado de: https://revistas.usal.es/index.php/11303743/article/view/teri.19616/20109

Sánchez, Gómez, Valero, De la Cruz, Belando, \& Moreno. (2019). Achieving greater sportsmanship and decreasing school violence through responsibility and sport practice. Journal of Sport Psychology.

Valdés, Á. A., Tánori, J., Sotelo, T. I., \& Ochoa, J. A. (2018). Prácticas docentes, clima social, seguridad escolar y violencia entre estudiantes. Magis. Revista Internacional de Investigación En Educación, 10(21), 109. doi:10.11144/Javeriana.m10-21.pdcs

Valle, M., Muñoz de la Torre, A., Robles, R., Vega, M. G., Flores, M. E., \& González, G. (2019). La violencia y acoso escolar en una escuela de Guadalajara, México. Revista Iberoamericana de Educación, 79. Recuperado de:

https://rieoei.org/RIE/article/view/3180/4010
GARCÍA-GARCÍA, Jesús Alberto, OSORNIA-RAMÓN, Larissa, RAMÍREZ-CERECERO, José Ricardo y MORÁNDELGADO, Gabriela. Violencia escolar y calidad de vida relacionada con la salud en niños de escuelas primarias. Revista de Educación Básica. 2020 